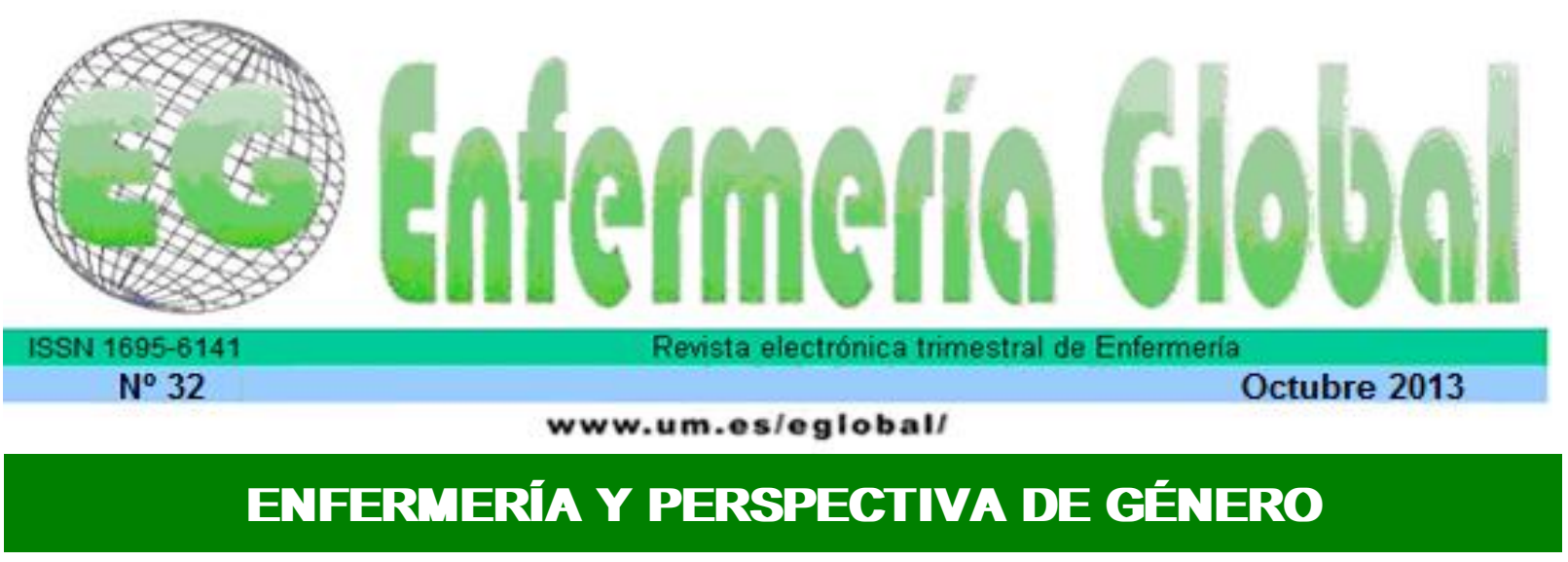

\title{
Características y evolución del patrón sexual de la mujer embarazada
}

Characteristics and evolution of pregnant women's sexual pattern

\section{${ }^{*}$ Rodríguez León, Marta **Ramón Arbués, Enrique}

\author{
*Enfermera especialista en Obstetricia y Ginecología. Hospital Universitario Miguel Servet. \\ **Enfermero especialista en Obstetricia y Ginecología. Hospital Universitario Miguel Servet. Profesor en \\ el Grado en Enfermería. Universidad San Jorge. Zaragoza. E-mail: ramonarbues@hotmail.com
}

\section{Palabras clave: Embarazo; sexualidad; coito; educación sexual \\ Keywords: Pregnancy; sexuality; coitus; sex education.}

\section{RESUMEN}

Objetivo: Analizar los cambios que se producen en la sexualidad de la mujer durante el embarazo.

Material y método: El estudio consistió en un análisis descriptivo transversal sobre una muestra de 74 embarazadas. Se recogieron datos de filiación, estudios, nivel socioeconómico y cultural, edad, duración de la convivencia, hábitos sexuales, satisfacción con respecto a su vida sexual y educación sexual recibida.

Resultados: El interés sexual en la embarazada se ve disminuido con el embarazo. En general, la frecuencia de coitos a la semana también se encuentra disminuida. Más de la mitad de las encuestadas encuentran su vida sexual satisfactoria, sin olvidar que una pequeña parte de ellas aumenta su interés y mejora su vida sexual. Prácticamente todas las encuestadas tiene frecuentes encuentros no genitales, éstos son muy deseados y se les da mucha importancia tanto por parte de las mujeres como de sus parejas.

Conclusiones: Una mayor educación sexual durante este periodo efectuada por profesionales formados en salud sexual puede mejorar la satisfacción de la gestante y su estado general de salud.

\section{ABSTRACT}

Aim: To evaluate changes in women's sexuality during pregnancy.

Methods: Cross-sectional study on a sample of 74 pregnant women. We collected data on educational, socio-economic and cultural level, age, duration of cohabitation, sexual habits, satisfaction with their sex life and sexual education received. 
Results: Women's sexual interest is decreased with pregnancy. Weekly frequency of intercourse is also decreased. Over $50 \%$ of respondents are satisfied with their sex life, even for a small part of them increases interest and improves their sex life during pregnancy. Almost all respondents have frequent no genitals meetings, these meetings are very desirable for themselves and for their partners.

Conclusions: Increasing sexual education during pregnancy performed by professionals trained in sexual health can improve the satisfaction of pregnant women and their overall health.

\section{INTRODUCCIÓN}

La persona es una mujer o un hombre desde su nacimiento, con sus atracciones, deseos y fantasías particulares. La historia de cada persona como ser sexuado se desarrolla y evoluciona a lo largo de la vida, está presente en todas las épocas de la misma y se encuentra íntimamente relacionada con aspectos psicológicos, sociales y culturales.

Con el paso de los años, el significado del término sexualidad ha sufrido muchos cambios. En la actualidad, la erótica (o conjunto de expresiones de la sexualidad humana) cumple diferentes funciones y persigue distintos objetivos. Las prácticas sexuales han dejado de ser simplemente una función en pro de la reproducción para convertirse en un aspecto complementario en el mantenimiento de la pareja.

Las relaciones sexuales satisfactorias contribuyen a una vivencia saludable de la sexualidad en su sentido más amplio, ayudando a mantener el equilibrio psicofísico del individuo y, por tanto, su estado general de salud ${ }^{1}$.

En 1996, Masters y Johnson publicaron los resultados de sus investigaciones acerca de la influencia del embarazo sobre la respuesta sexual, demostrando que esta época modificaba inexorablemente la sexualidad de la mayoría de sus participantes ${ }^{2}$.

En la sexualidad de la pareja entra en juego una tercera variable al confirmarse el embarazo. A partir de este momento, las relaciones sexuales de la pareja girarán en torno al desarrollo del feto.

Generalmente, el déficit de información suele inhibir las prácticas sexuales debido al miedo que se genera ante la posibilidad de provocar complicaciones, abortos o partos prematuros ${ }^{3}$.

Es indudable que el embarazo supone una situación crítica para la mujer desde el punto de vista físico, psíquico y afectivo. Incluso, la transición a la maternidadpaternidad puede ser vista como una crisis psicosocial ${ }^{4}$. La mayoría de las mujeres, y sus parejas, sufren modificaciones en su patrón habitual de comportamiento sexual. En la época del embarazo, la evidencia disponible se inclina hacia una disminución del interés sexual, de la frecuencia de coitos, de la capacidad orgásmica y de la satisfacción sexual de la gestante ${ }^{5,6}$.

Algunos estudios especulan sobre la conveniencia de la actividad sexual durante el embarazo y sus consecuencias ${ }^{7}$. Creencias populares y tabúes hacen que, en algunos casos, las gestantes y sus parejas tomen determinaciones sobre su comportamiento sexual de manera equivocada ${ }^{3,8}$.

El objetivo de este trabajo es conocer, en las gestantes de nuestro medio, los cambios que se producen en las distintas dimensiones que comportan la sexualidad de la mujer, comparándolas con el estado previo al embarazo. 


\section{MATERIAL Y MÉTODOS}

Se realizó un análisis descriptivo transversal sobre una muestra de 74 gestantes atendidas en los meses de mayo a julio de 2012 en el Hospital Universitario Miguel Servet de Zaragoza. La población de estudio la formaron aquellas mujeres mayores de 16 años, sin contraindicación médica u obstétrica para algún tipo de práctica sexual y que aceptaron participar de manera voluntaria. Las participantes fueron captadas en su tercer trimestre de la gestación mediante muestreo consecutivo, solicitándoles la cumplimentación de un cuestionario que incluía: variables sociodemográficas de la gestante y su pareja, variables obstétricas y ginecológicas, variables relacionadas con su educación sexual, variables sobre hábitos sexuales y grado de satisfacción con su sexualidad. Estas últimas variables a través de una modificación del cuestionario de comportamiento sexual utilizado con anterioridad por Piñero et al. ${ }^{9}$ en una muestra similar.

El análisis de los datos se realizó mediante tablas de contingencia (test de Jicuadrado de Pearson) a través del software estadístico Epidat 3.1. El nivel de significación aceptado se situó en $p<0,05$.

\section{RESULTADOS}

A continuación se detallan las características de la muestra (Tabla 1).

Tabla 1. Características de la muestra. Análisis descriptivo de variables.

\begin{tabular}{|l|l|r|r|}
\cline { 3 - 4 } \multicolumn{2}{c|}{} & № & Porcentajes \\
\hline \multirow{3}{*}{ Edad de la } & $16-25$ & 8 & $(\%)$ \\
\hline Embarazada & $26-30$ & 13 & 10,81 \\
& $31-35$ & 33 & 17,56 \\
& $>35$ & 20 & 44,59 \\
\hline \multirow{3}{*}{ Estudios de la } & Bachiller & 23 & 27,02 \\
\hline Embarazada & Formación & 18 & 31,08 \\
& profesional & 33 & 24,32 \\
& Universitario & 6 & 44,59 \\
\hline \multirow{4}{*}{ Profesión de la } & Ama de casa & 10 & 8,10 \\
Embarazada & Parada & 4 & 13,51 \\
& Estudiante & 54 & 5,40 \\
& Trabajadora & 32 & 72,99 \\
\hline Número de & No & 32 & 43,24 \\
\hline gestación & Primigesta & & 43,24 \\
& Secundigesta o & 42 & 56,76 \\
\hline
\end{tabular}

Las fuentes de información acerca de la sexualidad en la época del embarazo referidas por las participantes fueron diversas. Las fuentes más habituales fueron internet y las amistades. Al contrario, la fuente menos habitual fueron los profesionales sanitarios. Incluso alrededor de un $12 \%$ de las participantes refirió no haber recibido ni poseer ningún tipo de información al respecto. Figura 1. 
Figura 1. Fuentes de información sobre sexualidad en el embarazo. № de participantes y porcentaje.

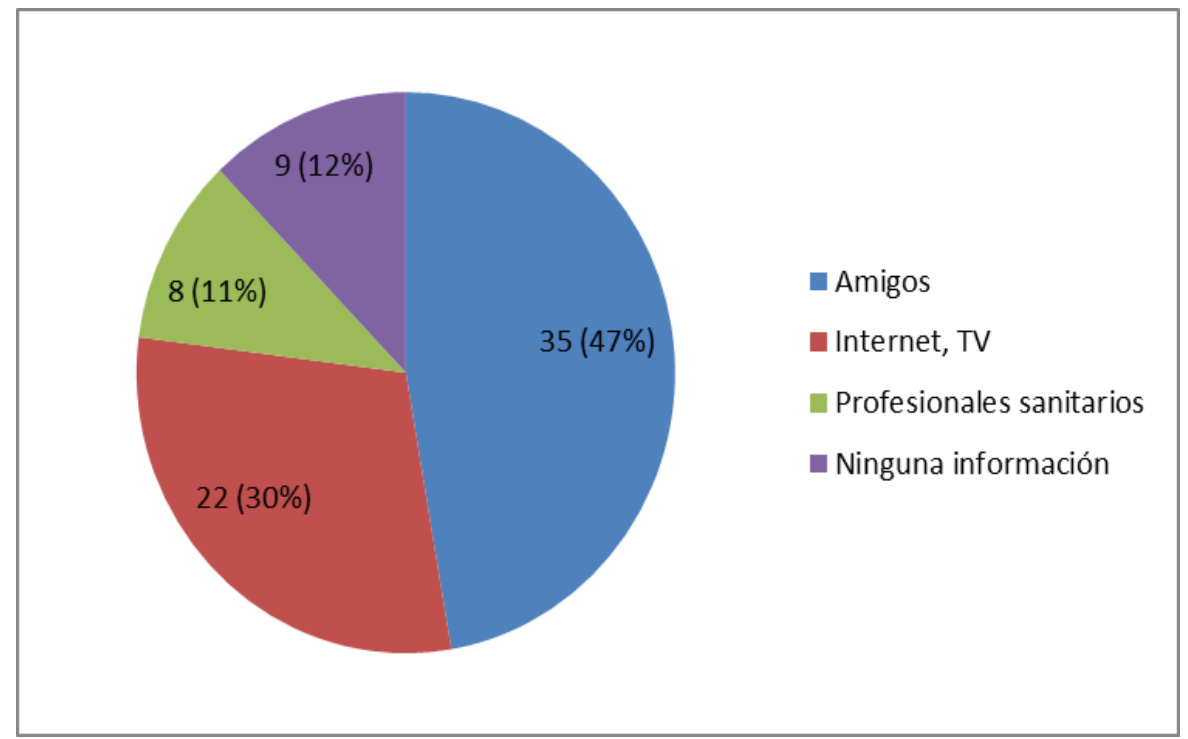

El interés sexual disminuyó significativamente en cada uno de los trimestres de la gestación $(p=0,0016)$, así como la frecuencia de los coitos $(p=0,000)$. Esta disminución fue más pronunciada en el segundo y tercer trimestre de la gestación. Figuras 2 y 3.

Figura 2. Interés sexual en la gestante por trimestres de gestación. № de participantes.

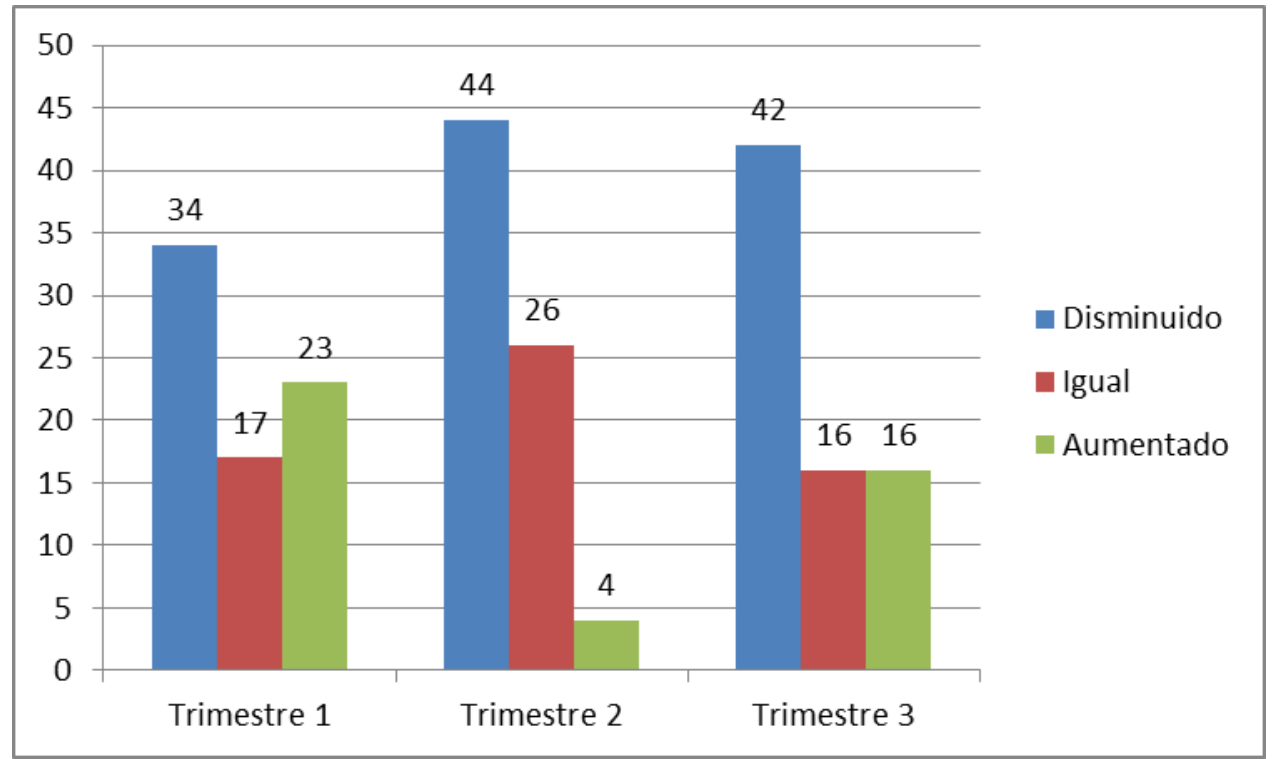


Figura 3. Frecuencia de coitos semanales antes y durante la gestación. № de participantes.

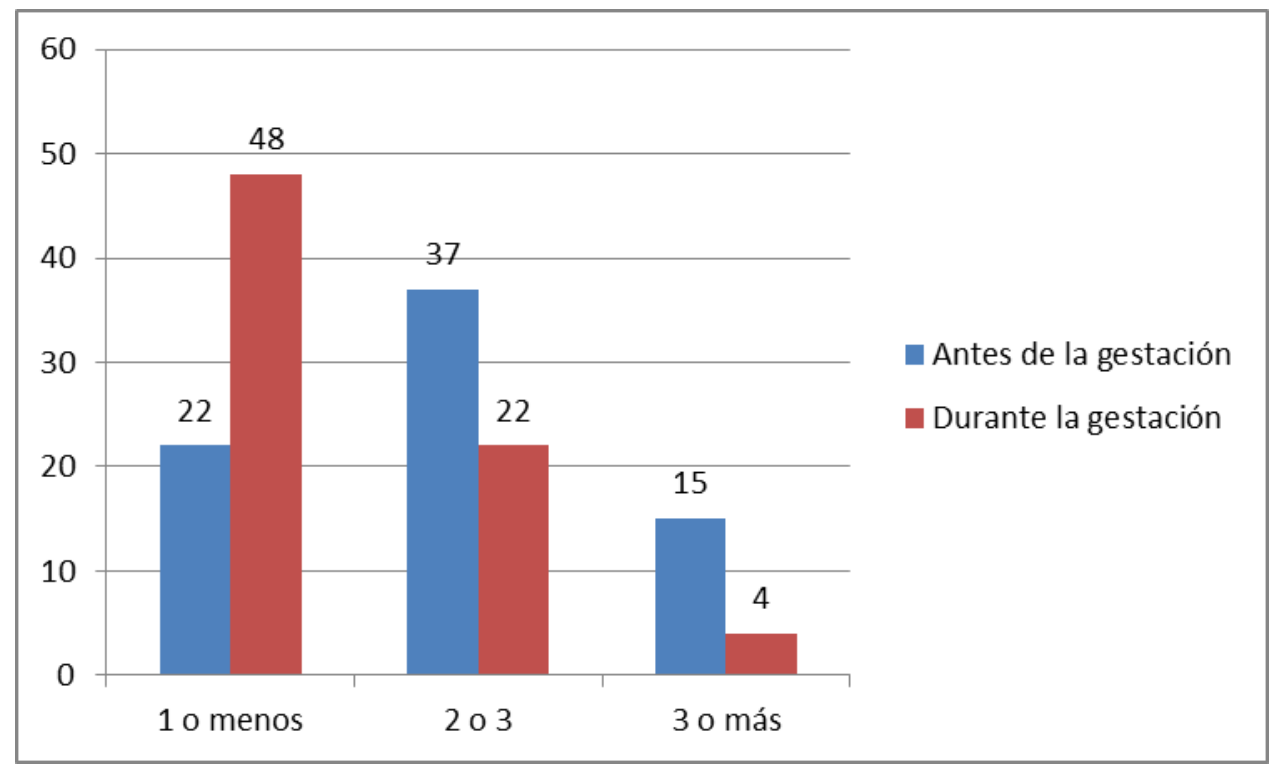

Al comparar las posiciones más empleadas en el coito se confirma que curiosamente perduraron las de "hombre encima" y "hombre debajo" por encima de otras basadas en la "lateralidad". En este sentido, no se observaron diferencias estadísticamente significativas en los patrones posturales de antes y después del embarazo $(p=0,88)$. Figura 4.

Figura 4. Posiciones más utilizadas en el coito antes y durante la gestación. № de participantes.

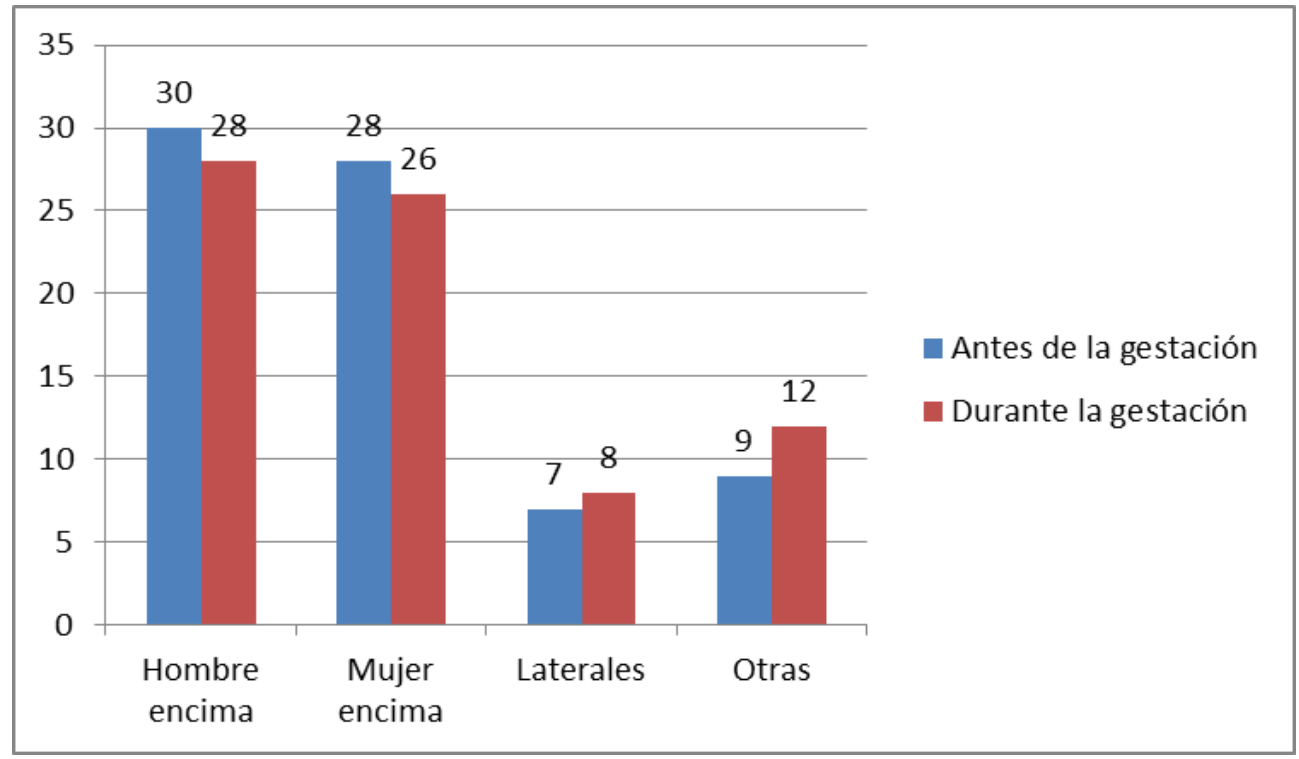


La auto-estimulación sexual aumentó para un $44,59 \%$ de las participantes en el segundo trimestre del embarazo y disminuyó en el primero y tercero, para un 58,10\% y un $75,67 \%$ respectivamente. En lo referente a las relaciones oro-genitales se observó una leve disminución de las mismas en el embarazo respecto a la época anterior al mismo. En cualquier caso, esta tendencia no pudo ser corroborada estadísticamente $(p=0,229)$. Figura 5.

Figura 5. Prácticas oro-genitales antes y durante el embarazo. № de participantes.

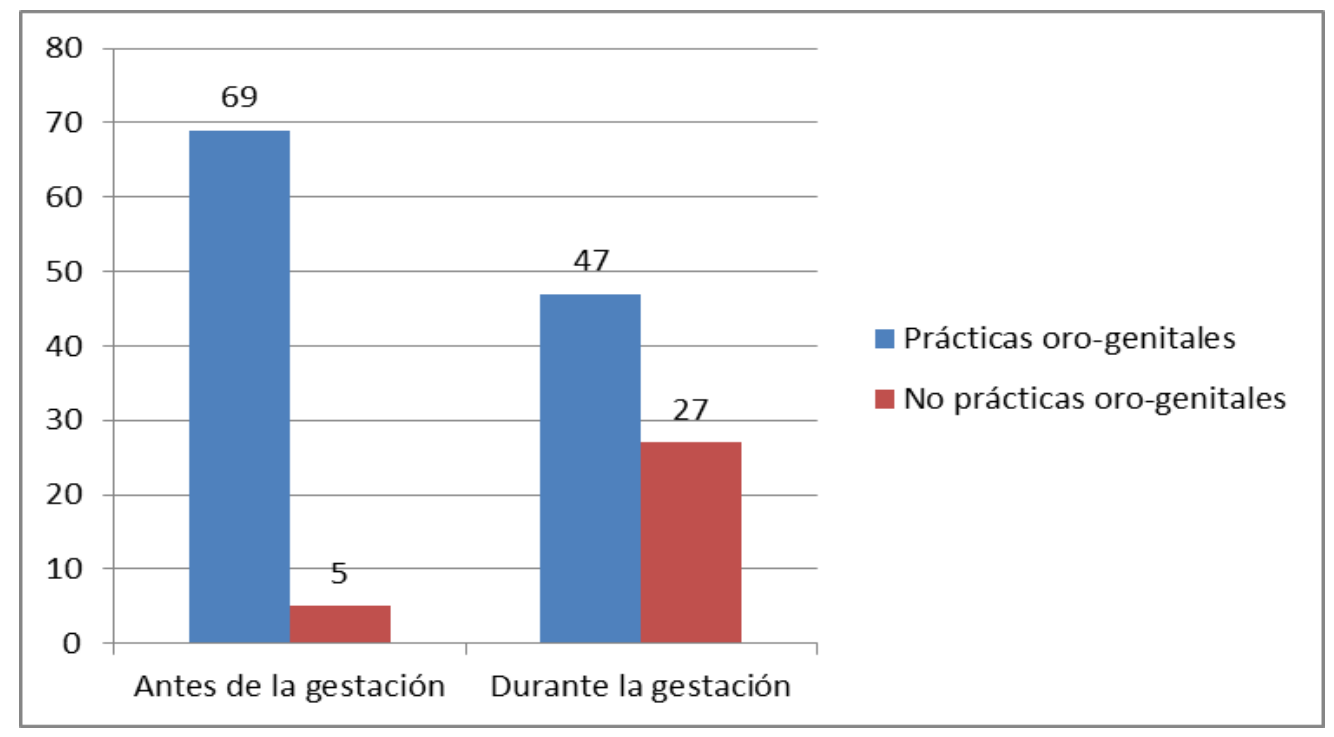

El embarazo disminuyó significativamente la satisfacción con la vida sexual $(p=0,000)$. En cualquier caso, en términos generales la mayoría de las mujeres siguieron encontrando su vida sexual satisfactoria también durante la gestación. Figura 6.

Figura 6. Satisfacción con la vida sexual antes y durante el embarazo. № de participantes.

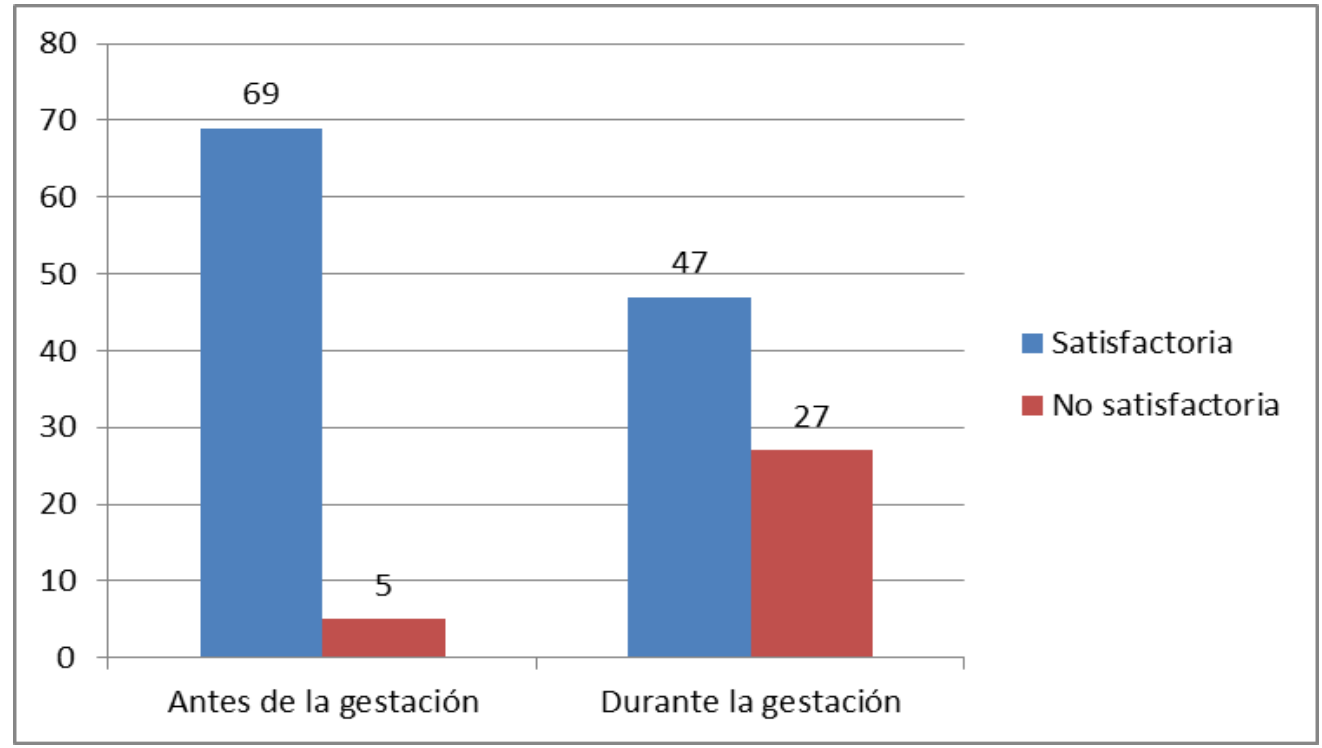


Las participantes alcanzaron con mayor dificultad el orgasmo en el segundo trimestre de la gestación $(p=0,000)$. No observándose diferencias estadísticamente significativas en el primer y el tercer trimestre. Figura 7.

Figura 7. Dificultad para la consecución del orgasmo a lo largo del embarazo. № de participantes.

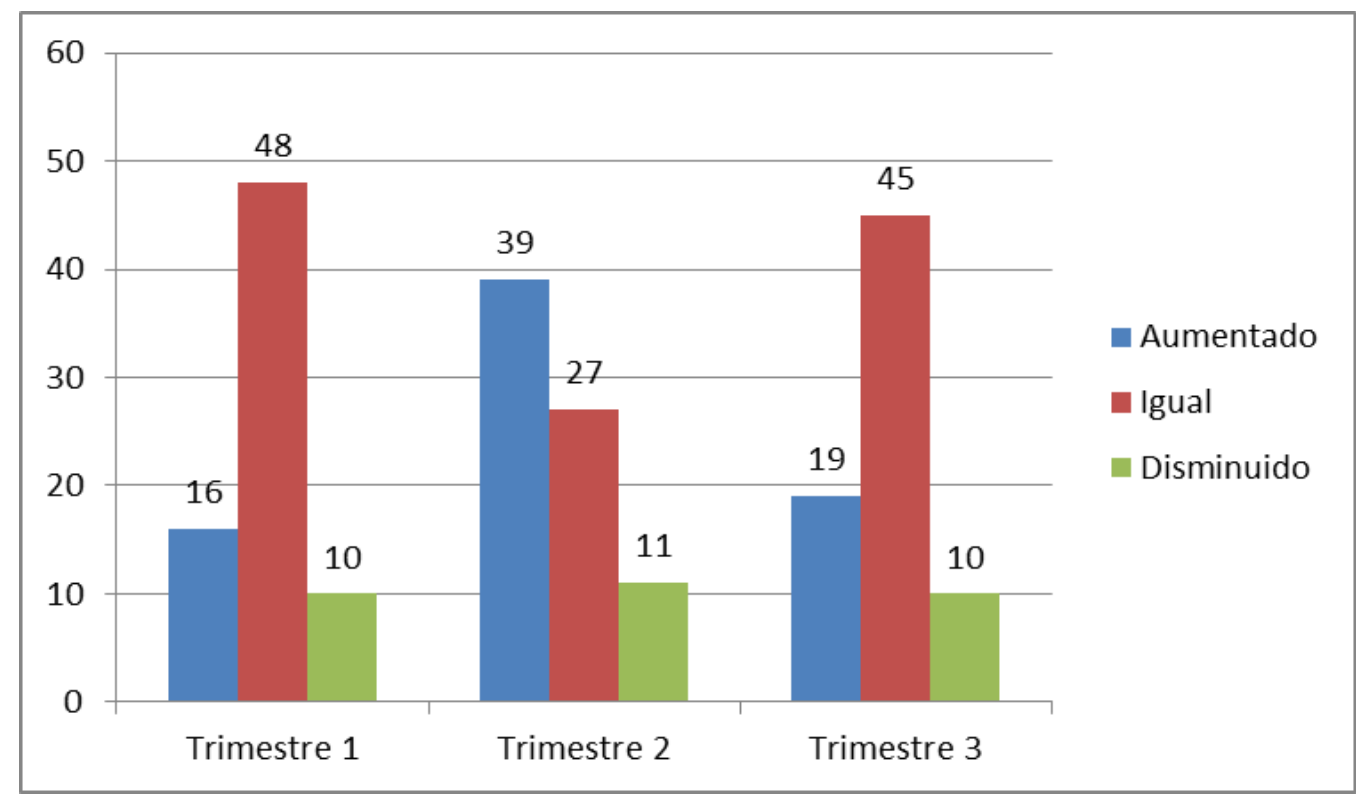

\section{DISCUSIÓN}

El interés sexual de la embarazada se ve disminuido en el primer trimestre, pero es en el segundo y tercero cuando más marcada se hace esta disminución. Estos resultados coinciden en la tendencia pero difieren en la magnitud de los resultados obtenidos por Pérez Sanz et al. ${ }^{1}$ quienes observaron un descenso más llamativo llegando a valores del $75,5 \%$ de pérdida de interés sexual en embarazadas en el tercer trimestre.

Al igual que lo observado por otros autores con anterioridad ${ }^{10,11,12}$, el número de coitos sigue una tendencia descendente a lo largo del embarazo, siendo más patente este descenso en el segundo y tercer trimestres. La dificultad para alcanzar el orgasmo es levemente superior en el segundo trimestre, cediendo ligeramente ésta en el tercero. Sin embargo, en otras investigaciones se observa que esta dificultad persiste hasta el final del embarazo ${ }^{13,14}$.

En nuestra muestra, el número de relaciones oro-genitales sufre un descenso durante el embarazo, aunque mucho menor si lo comparamos al de otras investigaciones ${ }^{15,16}$.

En este estudio, encontramos una mayor proporción de mujeres que se autoestimulan con respecto a otros trabajos revisados. Además la frecuencia de la misma a lo largo de la gestación se modifica en menor medida ${ }^{14,17}$.

Se observa que, en general, las relaciones sexuales sufren una disminución a lo largo del embarazo (en el número de coitos, orgasmos, auto-estimulación...) pero, a pesar de ello, más de la mitad de las gestantes se encuentran satisfechas y conformes con su vida sexual. 
A la luz de estos resultados, parece necesario incidir en la importancia de una mayor educación sexual durante este periodo por parte de profesionales formados en salud sexual. Más aún si tenemos en cuenta que una gran parte de los conocimientos sobre sexualidad de las embarazadas se nutren de las aportaciones de amigos, internet, etc., y no de personal sanitario cualificado. En esta línea, se ha demostrado que un mejor nivel educacional disminuye hasta en un $40 \%$ el riesgo de presentar disfunciones sexuales antes, durante y después del embarazo. Por otra parte, una adecuada educación sexual durante la época del embarazo puede ayudar a desmitificar los miedos maternos a causar lesiones fetales y a disfrutar de un embarazo y una sexualidad más plena y placentera.

\section{CONCLUSIÓN}

De forma general, las gestantes de nuestra muestra se muestran satisfechas con la vivencia de su sexualidad. Sin embargo, se objetiva una disminución del deseo y las prácticas sexuales durante esta época. Esta disminución es más marcada durante el tercer trimestre de la gestación.

Parece recomendable un esfuerzo educativo por parte de los profesionales sanitarios con el objeto de desmitificar miedos de las embarazadas y sus parejas.

\section{BIBLIOGRAFÍA}

1. Pérez Sanz P, Palacios Gil-Antuñano $S$. Aspectos de la sexualidad en la embarazada deducidos de un estudio de 100 embarazos. Progr. Obstet. Ginecolog. 1982;25(5):323-31.

2. Masters WH, Johnson VE. (1978) Respuesta Sexual Humana. Buenos Aires: InterMédica.

3. Bartellas E, JM Crane, M Daley, KA Bennett, D Hutchens. (2000). Sexuality and sexual activity in pregnancy. BJOG Aug. 2000;107(8):964-8.

4. Byrd JE, Hyde JS, DeLamater JD, Plant EA. Sexuality during pregnancy and the year postpartum. J Fam Pract. 1998 Oct; 47(4):305-8.

5. Pérez-López FR, Goicoechea J, Martínez Casamayor MF. Comportamiento sexual de la embarazada. Progr. Obstet. Ginecol. 1987;30(6):363-7.

6. Aslan G, Aslan D, Kizilyar A, Ispahi C, Esen A. A prospective analysis of sexual functions during pregnancy. Int J Impot Res. 2005 Mar-Apr:17(2);154-7.

7. Tejerizo López LC, Teijelo Déilos A, Moro Egido J, Sánchez Sánchez MM, García Robles RM, Cardeñoso Ahedo L et al. Sexualidad y parto pretérmino. Toko-Gin Pract. 1999;58(4):197-202.

8. Blümel M, Binfa L, Cataldo P, Carrasco A, Izaguirre H, Sarrá S. Índice de la función sexual femenina: Un test para evaluar la sexualidad de la mujer. Rev. Chil. Obstet. Ginecol. 2004;69(2):118-25.

9. Piñero Navero S, Alarcos Merinio G, Arenas Orta MT, Jiménez Iglesas $V$, Ortega Ballesteros EM, Ramiro Figueroa MJ. Características y modificaciones de la sexualidad durante el embarazo. NURE Inv. (Revista en Internet) 2011 Ene-Feb. (1104-2013); 8(50). Disponible en: http://www.fuden.es/FICHEROS_ADMINISTRADOR/ORIGINAL/NURE50_original_modifi $\underline{\text { sex.pdf }}$

10. Chang SR, Chen KH, Lin HH, Yu HJ. Comparison of overall sexual function, sexual intercourse/activity, sexual satisfaction, and sexual desire during the three trimesters of pregnancy and assessment of their determinants. J Sex Med. 2011 Oct;8(10):2859-67. 
11. Erol B, Sanli O, Korkmaz D, Seyhan A, Akman T, Kadioglu A. A cross-sectional study of female sexual function and dysfunction during pregnancy. J Sex Med. 2007 Sep;4(5):1381-7.

12. Corbacioglu Esmer A, Akca A, Akbayir O, Goksedef BP, Bakir VL. Female sexual function and associated factors during pregnancy. J Obstet Gynaecol Res. 2013 Jun;39(6):1165-72.

13. Sueiro E, Gayoso P, Perdiz C, Doval JP. Sexualidad en el embarazo: Vivencia que tiene la mujer de la suya propia y de la de su pareja. Revista Iberoamericana de Fertilidad. 1995;12(6):35-41.

14. Sueiro E, Gayoso P, Perdiz C, Doval JP. Sexualidad y embarazo. Aten Primaria. 1998;22:340-6.

15. López Olmos J. Sexualidad en el embarazo. Estudio Prospectivo. Progr. Obstet. Ginecol. 1998;31(4):219-30.

16. Serati M, Salvatore S, Siesto G, Cattoni E, Zanirato M, Khullar V et al. Female sexual function during pregnancy and after childbirth. J Sex Med. 2010 Aug;7(8):278290.

17. Pauleta JR, Pereira NM, Graça LM. Sexuality during pregnancy. J Sex Med. 2010 Jan;7(1):136-42. 